[Supporting Information]

\title{
Perforated Vesicles of ABA Triblock Copolymers with On/Off-Switchable Nanopores
}

\author{
Ting-Ya Wang ${ }^{1}$, Heng-Kwong Tsao ${ }^{2, *}$ and Yu-Jane Sheng ${ }^{1, *}$ \\ ${ }^{1}$ Department of Chemical Engineering, National Taiwan University, Taipei 10617, \\ Taiwan, ROC \\ ${ }^{2}$ Department of Chemical and Materials Engineering, National Central University, \\ Taoyuan 32001, Taiwan, ROC
}


Leaking simulations of vesicles with different $a_{A B}$

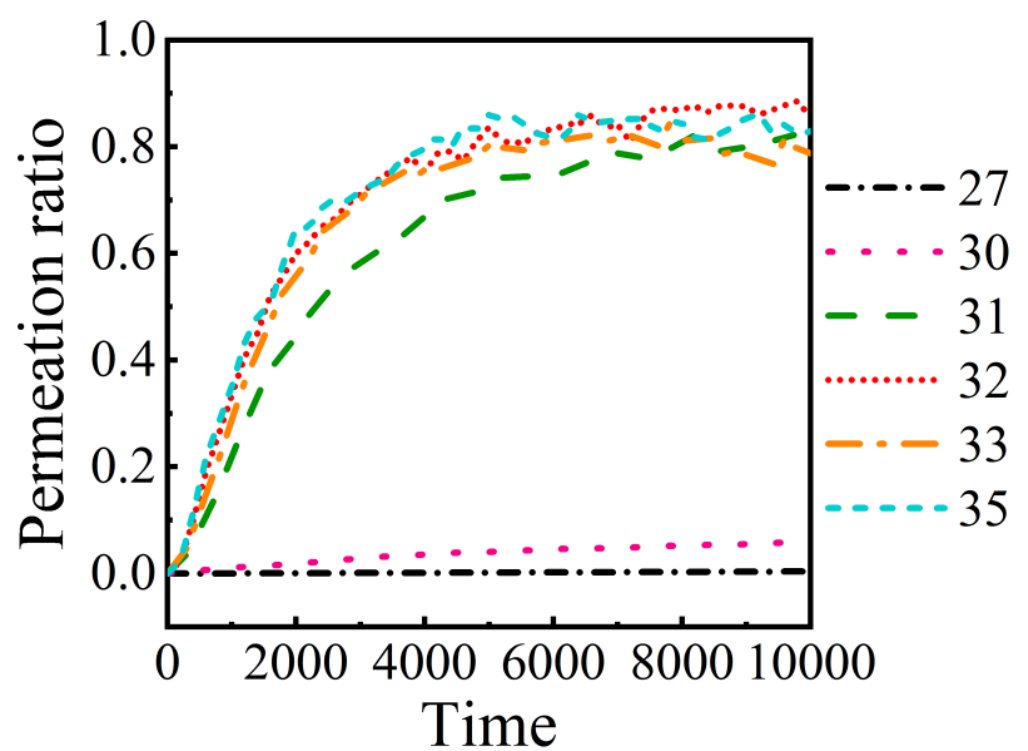

Figure S1. The time evolution of the permeation ratio for different values of $a_{A B}$ at $k_{\theta}=5$ and $a_{A W}=26$. 